\title{
Frustrated magnetic vortices in a triad of permalloy rings: Magneto-optical Kerr effect, magnetic force microscopy, and micromagnetic simulations
}

\author{
V. Rose, ${ }^{1,2}, *$ K. Buchanan, ${ }^{2}$ S.-H. Chung, ${ }^{2}$ M. Grimsditch, ${ }^{2}$ V. Metlushko, ${ }^{3}$ A. Hoffmann, ${ }^{2}$ V. Novosad, ${ }^{2}$ \\ S. D. Bader, ${ }^{2}$ and H. Ibach ${ }^{1}$ \\ ${ }^{1}$ Institute for Surfaces and Interfaces (ISG 3) and Center of Nanoelectronic Systems for Information Technology (CNI), \\ Research Center Jülich, D-52425 Jülich, Germany \\ ${ }^{2}$ Material Science Division and Center for Nanoscale Materials, Argonne National Laboratory, Argonne, Illinois 60439, USA \\ ${ }^{3}$ Electrical and Computer Engineering, University of Illinois at Chicago, Chicago, Illinois 60612, USA \\ (Received 19 August 2005; revised manuscript received 26 October 2005; published 28 March 2006)
}

\begin{abstract}
The field dependent magnetization of three mutually touching permalloy rings were investigated by means of the magneto-optical Kerr effect, magnetic force microscopy, and micromagnetic simulations. Each ring has a width of $0.2-1.8 \mu \mathrm{m}$, an outer diameter of $4 \mu \mathrm{m}$, and a thickness of $17 \mathrm{~nm}$. Decreasing an applied magnetic field from saturation leads to the nucleation and annihilation of magnetic vortices, leaving at least one ring in a magnetically frustrated state. The properties of the magnetization reversal strongly depend on the ring width and on the direction of the applied field. Multiple complex reversal paths with vortexlike magnetization configurations are found.
\end{abstract}

DOI: 10.1103/PhysRevB.73.094442

PACS number(s): 75.75.+a, 75.60.Ch, 75.60.Jk

\section{INTRODUCTION}

Understanding and controlling the magnetization of $\leqslant 1 \mu$ scale magnetic structures is of considerable basic interest and of huge importance for technological applications, such as magnetic random access memory, ${ }^{1,2}$ and data storage. ${ }^{3,4} \mathrm{~A}$ matter of particular interest is the accurate control of the switching between magnetic states. Each structure should exhibit an easily accessible plane magnetic anisotropy, i.e., in addition to the remanent state, a second well-defined state is required to enable the implementation of binary bits. Further applications need fast and reproducible switching and the magnetic states should be stable and reproducible. Coherent reversal of single domain states seems to be ideal. In the last years, several structures such as rectangles, ${ }^{5}$ ellipses, ${ }^{6}$ pyramids, ${ }^{7}$ and needles ${ }^{8}$ have been investigated as potential candidates. It appears, however, that it is difficult to control their magnetization. Due to dipole interactions, the direction of the magnetization changes in the proximity of the boundaries of the structures and forms small edge domains that are sensitive to the shape and the roughness of the structures. ${ }^{9,10}$ One possibility to overcome these problems is the use of discs. ${ }^{9,11}$ Even more stable magnetization states arise from ferromagnetic rings. ${ }^{12}$ Generally, they can be reversibly switched between two stable magnetic states in an applied magnetic field. At saturation, the ring shows a single domain state, where all spins are aligned to the field. In the course of field relaxation, the ring switches into a bidomain state referred as an onion state. ${ }^{13}$ Here, the magnetization follows the circumference of the ring with opposite senses of circulation in the two halves of the ring. Two opposite head-tohead Néel domain walls are formed generating a stray field. Calculations of head-to-head domain wall structures in magnetic strips and rings predict either "transverse" walls with magnetization at the center of the wall directed transverse to the strip axis or "vortex" walls where the magnetization forms a small vortex at the center of the wall. ${ }^{14,15}$ After re- moving the field, the onion state remains stable at remanence. ${ }^{13}$ When a reverse field is applied, ideally both domain walls should move in the same direction around the ring. However, due to imperfections in patterned rings, which always exist in real experiments, one wall will be more strongly pinned than the other. Consequently, the two walls are annihilated when the depinned wall reaches the pinned one and a vortex state is created, which is characterized by a flux-closure magnetization with the zero stray field. ${ }^{12}$ The vortex state is stable for a certain magnetic field strength and subsequently annihilates at higher reverse fields. Hence, the ring switches first from the onion state to the vortex state and than to the opposite onion state. ${ }^{16}$ Depending on the geometrical ring parameters (thickness $t$, width $W$, outer diameter $D$ ), the spin switching in ferromagnetic rings may vary. Thin rings willingly exhibit a direct onion to reverse onion transition, whereas thick rings show multistep switching. ${ }^{17}$

While the study of single, isolated ferromagnetic rings has attracted attention because of their fascinating properties, planar arrangements of several rings have barely been studied. Nevertheless, interactions between rings can have a significant effect on the reversal process. Dipolar domain wall coupling is important for separations that are approximately $\leqslant 1$ ring diameter ${ }^{18}$ and exchange coupling will also have an influence for rings in direct contact, as demonstrated for connected chains of dots and rings. ${ }^{19-21}$

In the present work, we study the interaction of tri-ring structures where three rings touch each other in a triangular layout. This system is of interest because magnetically frustrated states can arise due to their geometric arrangement. When two rings in direct contact interact, it is expected that their vortex states will have opposite chirality. However, for an arrangement of three mutually interacting rings, we expect a spin frustration between competing magnetic states. 


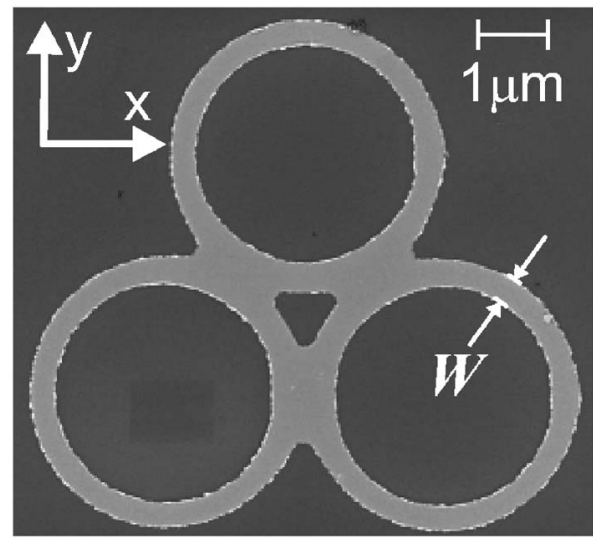

FIG. 1. Scanning electron micrograph of a triad of permalloy rings. The ring width is $W=0.4 \mu \mathrm{m}$ and the associated coordinate system is shown.

\section{EXPERIMENTAL}

We have fabricated arrays of tri-ring permalloy ( $\mathrm{NiFe}$ alloy) structures by means of electron-beam lithography (using a Raith Model 150 at $10-\mathrm{keV}$ beam energy). The rings have widths $W=0.2-1.8 \mu \mathrm{m}$, outer diameter $D=4 \mu \mathrm{m}$, and thickness $t=17 \mathrm{~nm}$. The tri-ring structures were drawn in a 116-nm-thick spin-coated layer of polymethyl methacrylate (PMMA) resist on a thermally oxidized $\mathrm{Si}(100)$ substrate. After developing the resist pattern [with methyl iso butyl ketone (MIBK):isopropyl alcohol (IPA) 1:3], the permalloy layer was grown by electron-beam evaporation in a high vacuum. The desired structure was then obtained after a subsequent chemical liftoff process. Figure 1 shows a scanning electron microscopy (SEM) image of the tri-ring structure with $W=0.4 \mu \mathrm{m}$, as well as the associated coordinate system. The structure is geometrically invariant upon rotation by $60^{\circ}$ in a magnetic field. The field dependent magnetization was investigated utilizing magnetic force microscopy (MFM) and transverse magneto-optical Kerr effect (MOKE) measurements. In order to facilitate MOKE measurements, $100 \times 100 \mu \mathrm{m}^{2}$ arrays of identical tri-ring structures were fabricated for each ring width. The MFM tip was coated with a CoCr alloy magnetized along the tip direction. Thus, a field that is applied in plane will not change the MFM contrast other than insofar that it changes the domain structure.

In order to gain insight into the features in the MOKE hysteresis loops and the MFM images, we performed micromagnetic simulations using the LLG MiCROMAGNETICS SimUlator software. ${ }^{22}$ This program solves the LandauLifshitz-Gilbert equations ${ }^{23,24}$ using finite differences for exchange energies and fields, as well as boundary elements for magnetic self-energies and fields. The structure was discretized in a two-dimensional (2D) mesh with cell size of $15 \times 15 \mathrm{~nm}^{2}$. The parameters of the magnetically soft permalloy used in the simulations are the exchange constant $C=2 A=2.6 \times 10^{-6} \mathrm{erg} / \mathrm{cm}$, and the saturation magnetization $M_{s}=800 \mathrm{emu} / \mathrm{cm}^{3}$, resulting in an exchange length of $R_{0}=C^{1 / 2} / M_{s}=20.2 \mathrm{~nm}$. Crystalline anisotropy was neglected.
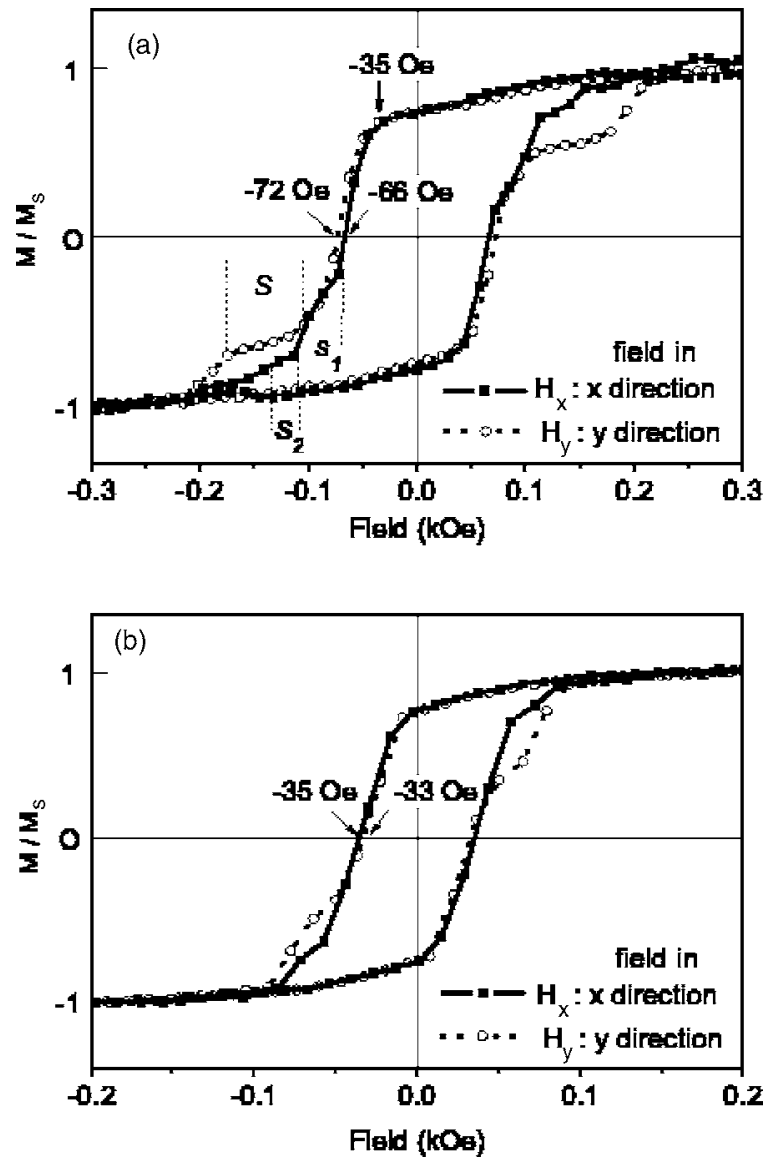

FIG. 2. MOKE hysteresis loops for $W=0.19 \mu \mathrm{m}$. The loops for the $H_{y}$ field applied in the $y$ direction (filled squares) exhibit a pronounced step feature with broadness $S$, loops with the $H_{x}$ field in the $x$ direction (open circles) show only weak steps $s_{1}$ and $s_{2}$. (b) For $W=0.89 \mu \mathrm{m}$, the broadness of the steps and the coercivity are decreased.

\section{RESULTS AND DISCUSSION}

\section{A. Magneto-optical Kerr effect and simulations}

In Fig. 2(a), we present MOKE measurements for an array of tri-rings with $W=0.19 \mu \mathrm{m}$. The filled squares correspond to loops for the applied magnetic field in the in-plane $x$ direction $\left(H_{x}\right)$, which is along the midpoints of two of the rings (Fig. 1). The open circles are for loops with the field in the $y$ direction $\left(H_{y}\right)$, which is tangential to the connection between two of the rings (Fig. 1). Reducing the field from positive saturation, the signal for both cases decreases abruptly at a negative critical field of -35 Oe. The coercivities are 66 and 72 Oe for $H_{x}$ and $H_{y}$, respectively. The hysteresis loop shows two weak steps $\left(s_{1}, s_{2}\right)$ on either side for the $H_{x}$ case, but only one broad, but pronounced step feature $(S)$ for the $H_{y}$ case, which indicates that the reversal processes differ for the two cases. For a larger ring width $W=0.89 \mu \mathrm{m}$ [Fig. 2(b)] the coercivities are only 35 Oe and 33 Oe for $H_{x}$ and $H_{y}$, respectively. Both the broadness of the steps and the values of the coercivity are decreased relative to those in Fig. 2(a). First, we examine the coercivity in more detail. 


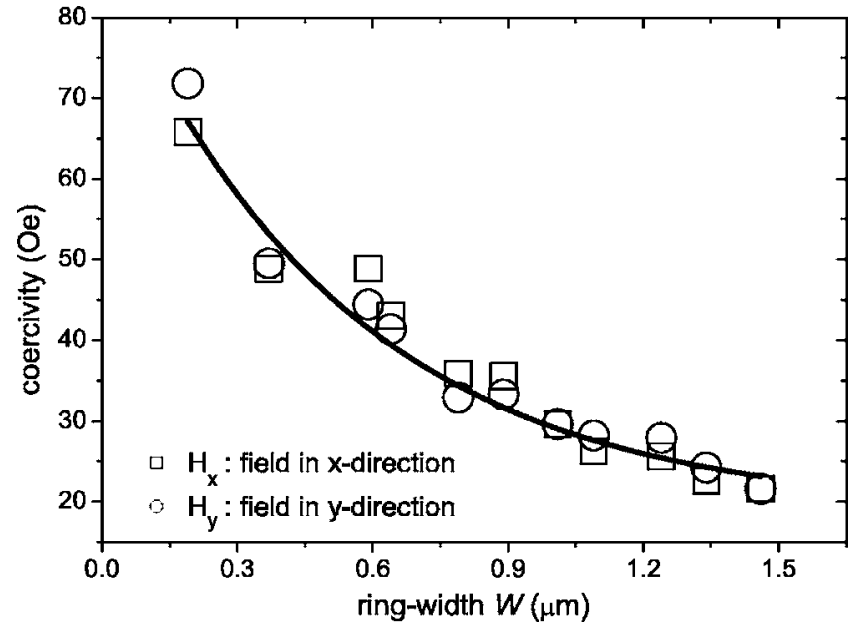

FIG. 3. Coercivity of tri-ring structures with different values of the ring width $W$. The data for the field in the $x$ direction (squares) and $y$ direction (dots) show an exponential decay.

Figure 3 shows the dependence of the coercivity for several tri-ring structures as a function of $W$. The values obtained for the $H_{x}$ and $H_{y}$ cases were fit by a single curve, which demonstrates that the direction of $H$ does not affect the coercivity. Increasing $W$ causes the coercivity to gradually decrease from $\sim 70$ Oe for $W=0.19 \mu \mathrm{m}$ to 20 Oe for $W=1.46 \mu \mathrm{m}$. In the wider rings, the spins are less strongly aligned to the circumference and can, therefore, twist more easily. Figure 4 shows a polar plot of the coercivity as a function of measurement angle for a particular tri-ring structure with $W=0.65 \mu \mathrm{m}$. The coercivity is independent of the direction of the applied field and exhibits an average value of $43 \pm 4$ Oe.

The appearance of pronounced steps is a notable feature of the hysteresis loops shown in Fig. 2. In the case of isolated single rings, such steps in the hysteresis loops were assigned to the formation and annihilation of a magnetic vor-

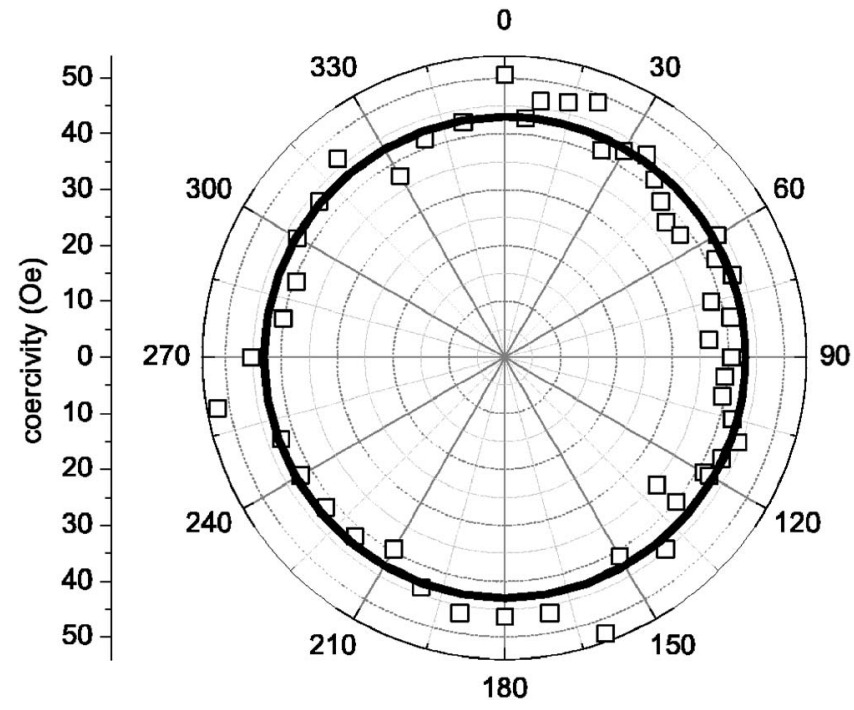

FIG. 4. The polar plot illustrates the independence of the coercivity on the direction of the applied field in the case of a structure with $W=0.65 \mu \mathrm{m}$. The average coercivity value is $43 \mathrm{Oe}$.

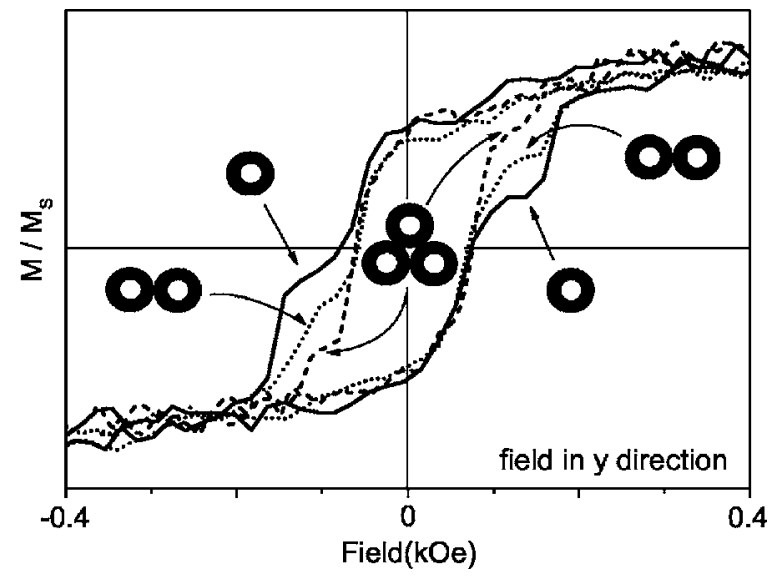

FIG. 5. MOKE hysteresis loops of single, double, and triple rings with field in the $y$ direction (diameter $D=4 \mu \mathrm{m}$, thickness $t=17 \mathrm{~nm}$, width $W=0.3 \mu \mathrm{m}$ ). As one goes from single- to doubleto tri-ring structures, the stability of the vortex state is reduced.

tex state. ${ }^{12}$ Also, single rings with the dimension of those in the tri-ring structures enter the vortex state. An example is given in Fig. 5, where we compare hysteresis loops of single, double, and triple rings with $W=0.3 \mu \mathrm{m}$ in an $H_{y}$ field. Characteristic steps can be obtained for all configurations. However, the graph shows very clearly that for an increasing number of connected rings, these steps are less pronounced. Hence, the stability of the vortex state is reduced.

In order to understand the origin of the steps in our triring case in more detail, we carried out micromagnetic simulations. In Fig. 6, we present a comparison between experimental MOKE and simulated hysteresis loops for a tri-ring structure (with $W=1 \mu \mathrm{m}$ ) in an $H_{y}$ field. The qualitative ex-

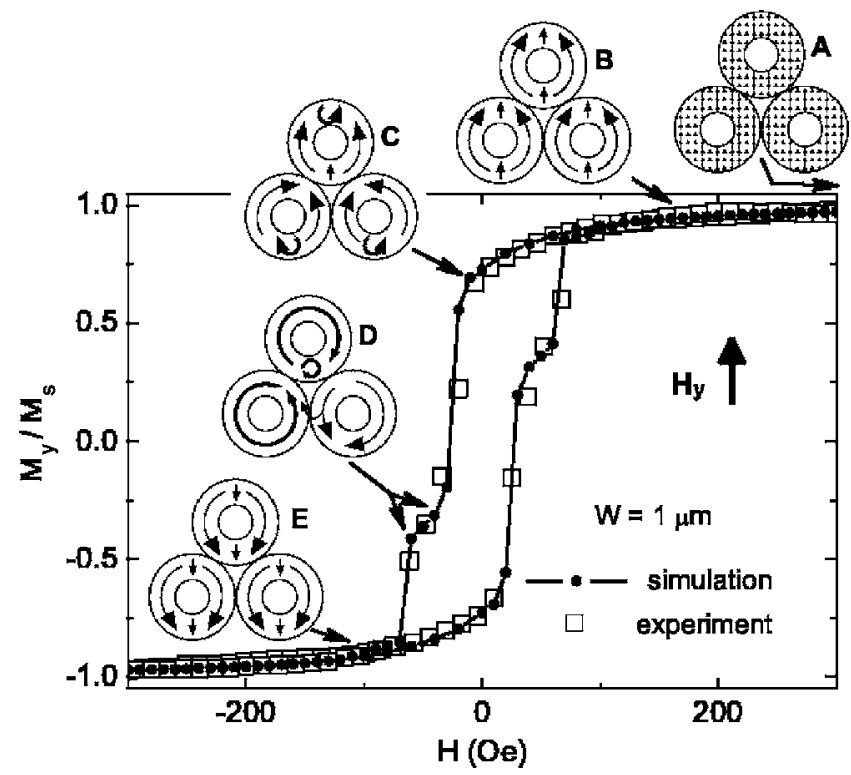

FIG. 6. Experimental hysteresis loop (squares) and micromagnetic simulations (dots) of a tri-ring structure with $W=1 \mu \mathrm{m}$ in a field in the $y$ direction. According to the simulations, the step feature is correlated to a magnetic state (see schematic (D) with two rings in vortexlike configurations, one with a vortex core, and the third ring experiences magnetic frustration. 
perimental shape is reproduced by the calculations. Starting at saturation, all rings exhibit a single domain state. Here, all spins are aligned to the external field (schematically shown as A). Relaxing the field leads to the formation of highremanence onion states with transverse head-to-head domain walls ${ }^{15}$ in the rings (B). At $H_{y}=-10$ Oe, three small vortices nucleate at the three extremities of the tri-ring structure along the field direction, forming vortex head-to-head domain walls ${ }^{15}(\mathrm{C})$. With the increasing field, these small vortices start to move clockwise (in the top and bottom-left rings) and anticlockwise (bottom-right ring). Thus, the whole spin configuration rotates along the circumferences of the rings. At $H_{y}=-30 \mathrm{Oe}$, where the hysteresis loop shows a transition, one ring switches to a vortex state (bottom left), a second ring remains in a frustrated onionlike state (bottom right), and a small vortex core arises inside of the top ring between the contact areas with the two other rings (D). The top ring shows a modified vortexlike magnetization that is frustrated by the small vortex core inside of this ring. Instead of having a vortex state confined to the top ring, a flux closure state can be traced around the main part of the top ring and then through the contact region between the bottom rings. In the following discussion, this complex magnetic configuration (D) will be referred to as the $H_{y}$-vortex state. Initially, the vortex core is located close to the contact area with the ring in the onion state. An increasing field leads then to a migration of the vortex core perpendicular to the field direction, until the vortex core reaches the contact area with the other ring. At $-60 \mathrm{Oe}$, the vortices inside of the two rings are simultaneously annihilated and disappear. For higher field values, all rings of the tri-ring structure exhibit an opposite onion state. Finally, at negative saturation, all rings exhibit a single domain state, inverse to configuration $\mathrm{A}$.

The same tri-ring structure shows a different reversal mechanism for an $H_{x}$ field (Fig. 7), in which the field is aligned along the midpoints of two of the rings. In this case, two different reversal paths were found in the simulation. As a starting condition, the rings exhibit a single domain state with perfect alignment of the spins at positive saturation (A). While relaxing the field, the bottom two rings form a quasionion state with no domain wall at their boundary and only one transverse head-to-head domain wall in each ring (B). The third ring exhibits a regular onion state. At $-20 \mathrm{Oe}$, four vortex cores appear in the former positions of the transverse head-to-head domain walls of the onion states $(\mathrm{C})$. While the top ring maintains its magnetization state, the small vortices of the bottom rings move to form a new vortex core at their mutual boundary, accompanied by an interring vortex in which all three rings participate (D). Between -80 and -100 Oe, only the vortex core at the boundary remains (E), while the rings themselves assume an onion state. In this region, the hysteresis loop shows a small step, which indicates that this vortex core possesses the highest stability on this path. For negative fields beyond $-100 \mathrm{Oe}$, the rings are again in a quasionion state, inverse to $\mathrm{B}$. On the reverse path, initially one vortex nucleates around the top ring $\left(\mathrm{A}^{\prime}\right)$. This vortex then dictates an opposite chirality of the vortices that appear in the other two rings. Consequently, magnetic frustration arises at their boundary. The tri-ring structure releases

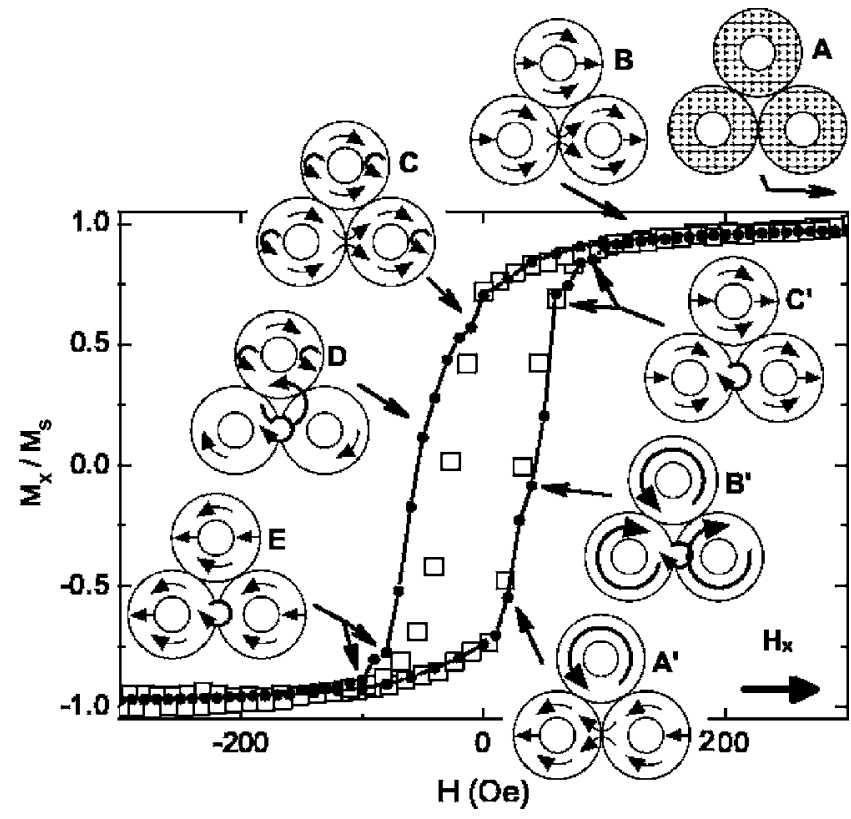

FIG. 7. Micromagnetic simulation (dots) and experimental data (squares) for the same tri-ring structure as shown in Fig. 6, but now with field in the $x$ direction. Two different reversal paths are found numerically but both ultimately produce a vortex core between the ring boundaries aligned to the field (see $\mathrm{E}, \mathrm{C}^{\prime}$ ).

this frustration by creating a vortex core at the boundary, while an overall flux closure state encircles the two rings $\left(\mathrm{B}^{\prime}\right)$. This magnetization configuration evolves into one with a vortex core at the boundary of two of the rings $\left(\mathrm{C}^{\prime}\right)$, similar to state $\mathrm{E}$ on the other path, referred to as the $H_{x}$-vortex core state.

It is remarkable that the micromagnetic simulations yield multiple paths with a small asymmetry of the simulated hysteresis loop. Most likely, this can be assigned to an insufficient saturation at the negative field. Consequently, the micromagnetic configuration might be slightly different at positive and negative saturation. This result also indicates that there may be multiple reversal paths that have only a small difference in energy. A number of intermediate states are observed in the simulations; however, these states are stable over small field ranges as compared to the $H_{y}$ reversal path. In the experimental hysteresis loops, the small steps that are related to the $\mathrm{E}$ and $\mathrm{C}^{\prime}$ are not resolved. This might be due to the fact that the MOKE data average over a range of tri-ring structures and imperfections might obscure the observation of such weak transitions or there may be a statistical distribution of switching fields or reversal paths. Nevertheless, these transitions can be detected experimentally for smaller $W$ values [e.g., in Fig. 2(a)]. For small $W$ values, the switching takes place at higher fields, the magnetic states show a higher stability, and there is an increased step broadness $S$, which is the difference between the creation and annihilation fields of the distinct vortex formations.

While the coercivity is invariant under the rotation of the applied field, the stability of the different vortex configurations is considerably influenced by the direction of the field. 


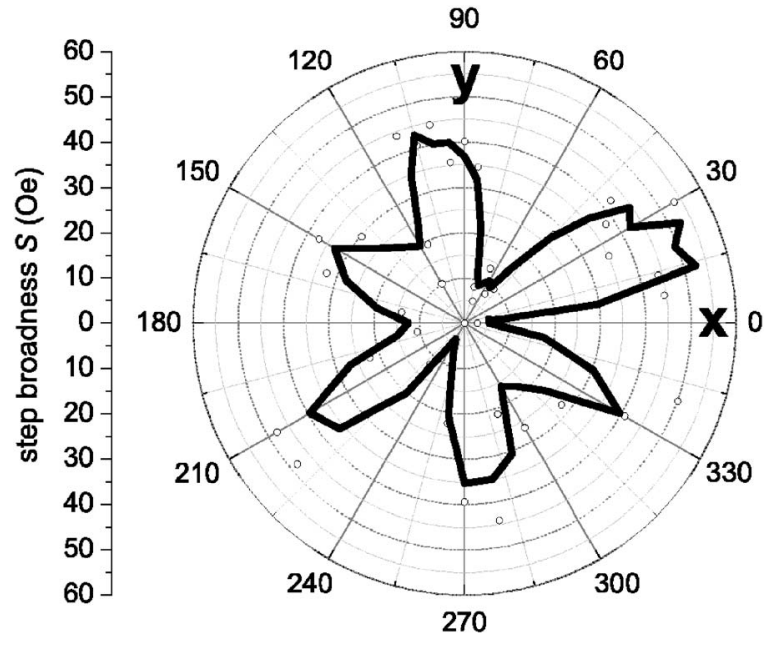

FIG. 8. Polar plot of the step broadness $S$ of the nucleation and annihilation of the vortex formations of a tri-ring structure $(W$ $=0.65 \mu \mathrm{m})$. The tri-ring structure is geometrically invariant upon rotation by $60^{\circ}$. The minima and maxima are related to the field directions $H_{x}$ and $H_{y}$, as discussed in the text.

Note that the steps in the hysteresis loops only appear for fields having an absolute value greater than the coercivity. In Fig. 8, the step broadness $S$ is plotted in polar coordinates as a function of the angle of the applied field. In the case where more than one transition was resolved in the hysteresis loops [like $s_{1}$ and $s_{2}$ in Fig. 2(a)], their absolute values were added together. The measurements illustrate the rotation invariance of the tri-ring structure, with $S$ exhibiting a maximum in the $H_{y}$ direction and at $60^{\circ}$ intervals, i.e., when the field is aligned along the axis of symmetry of the structure. These angles also correspond to minimal alignment of the contact areas with the field direction. The minima in the polar plot are correlated with the $H_{x}$ field. Unlike the case for isolated rings, domain walls can extend into neighboring rings and move across the contact areas of the rings. Such propagation is easier when the field is applied in the direction of a contact area (i.e., the $H_{x}$ case). Thus, if one contact area is aligned with the external field, the field can readily destroy the vortices and the vortex core.

Given the angular dependence of the step width, one might expect that the contact area between the rings is significant for the movement of domain walls across the rings. Figure 9 shows the coercivity of a tri-ring structures with $W=0.65 \mu \mathrm{m}$ with varied contact length $L$ between 0.59 and $1.35 \mu \mathrm{m}$. This was achieved by varying the center-to-center distance between the rings. The data shown were obtained by averaging the coercivities from angle dependent measurements for each $W$, as in Fig. 4, and the error bars are representative of the statistical error of each measurement. As mentioned previously, the coercivity is invariant with respect to the angle of the external field. Figure 9, however, suggests that the coercivity slightly decreases with increasing $L$. This can be explained by an enhanced interaction between the rings with increasing overlap. Generally, the domain wall moves around the perimeter of the rings. ${ }^{13}$ As a domain wall starts to move, its stray field can trigger the domain wall motion in an adjacent ring. Thus, the ring with the lowest

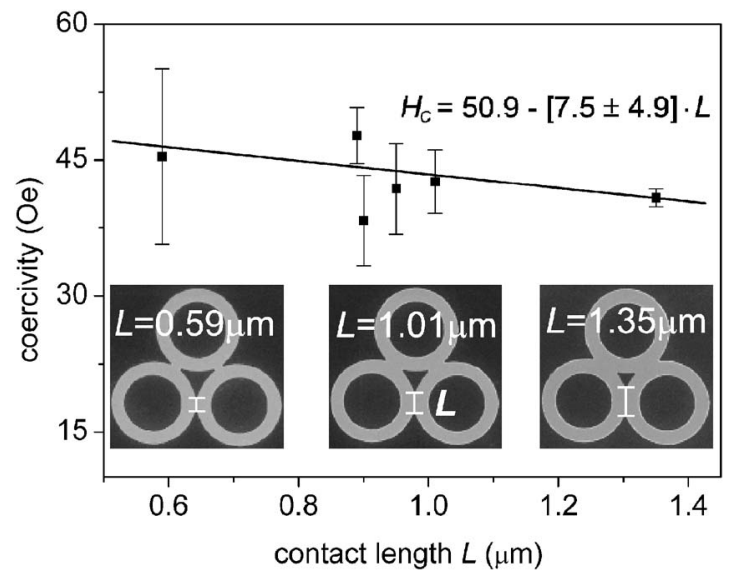

FIG. 9. The coercivity $H_{C}$ decreases with increasing ring contact length $L$. The linear regression of $H_{C}$ as a function of $L$ shows that only a negative fit gradient lies within error. The SEM images show some tri-ring structures $(W=0.65 \mu \mathrm{m})$ with varied $L$.

switching field can cause a cascade of switching inside of the tri-ring structure. Therefore, the switching field and the coercivity decrease with increasing interactions. Also, in the $H_{y}$-vortex state (D in Fig. 6), one ring is still in an onion state, i.e., this ring provides stray fields that add to the external field and facilitate the switching with increasing $L$. Similar stray fields are present in the $H_{x}$-vortex core state $\left(\mathrm{C}^{\prime}\right.$ in Fig. 7). Interestingly, the dispersion of the coercivity, i.e., the changes in the error bars, decreases with increasing $L$. This indicates that small irregularities in the array of identical tri-ring structures are more significant for small contact lengths.

The switching fields for the nucleation and annihilation of the $H_{y}$-vortex state as a function of $W$ are depicted in Fig. 10. The absolute value of the switching field of the nucleation and annihilation fields decreases with increasing $W$. Therefore, the $H_{y}$-vortex-state is energetically more stable in smaller rings. The tri-rings exhibit qualitatively the same

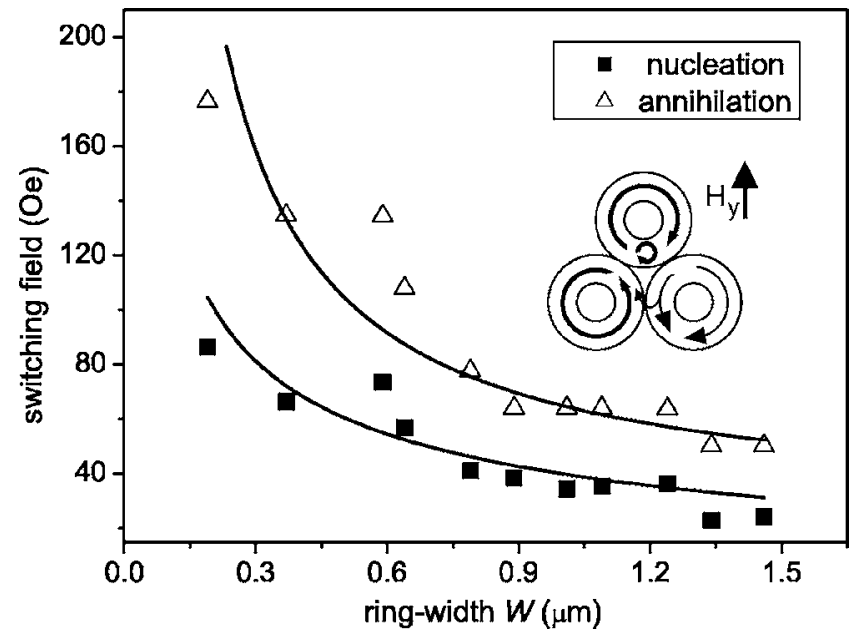

FIG. 10. Nucleation (squares) and annihilation (triangles) fields of tri-ring structures for different values of $W$ in an $H_{y}$ field. 

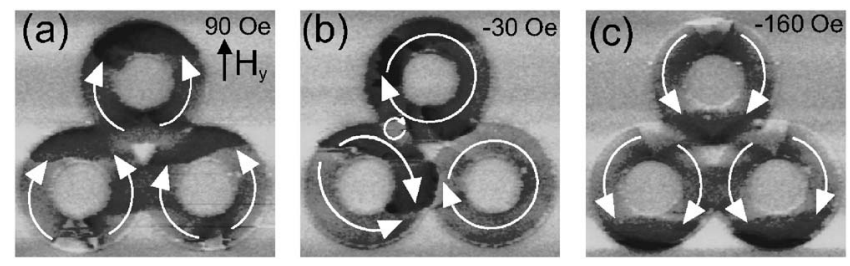

FIG. 11. MFM images in an $H_{y}$ field. Starting from saturation (a) at $90 \mathrm{Oe}$, all rings $(W=1 \mu \mathrm{m})$ are in an onion state. (b) At $-30 \mathrm{Oe}$, the lower right ring is in a vortex state, while the other rings show several domain walls. (c) Domain walls appear at the bottom and the top of the rings $(-160 \mathrm{Oe})$ indicating a reverse onion states of the rings.

behavior as isolated rings. In the case of single Co rings, it was also found that the narrower the rings are, the higher the vortex-to-onion switching field is ${ }^{25}$ Likewise, in $\mathrm{Ni}$ micro rings, the vortex state becomes more stable in smaller rings. ${ }^{26}$ Generally, in rings with small $W$, there is on average a higher alignment of the spins parallel to the edge of the ring that suppresses the nucleation of a reverse domain and, therefore, increases the switching fields.

\section{B. Magnetic force microscopy}

In order to test the micromagnetic simulations, we performed MFM imaging of the domain configurations in the tri-ring structures. However, tip-sample interactions make it difficult to map the intermediate magnetic states of the soft permalloy tri-rings near the coercive field. Nevertheless, the imaging of several predicted magnetic states was achieved.

Figure 11(a) shows a MFM image after reducing the applied $H_{y}$ field from saturation to 90 Oe. $W=1 \mu \mathrm{m}$, as in the simulations. The light (bottom of the rings) and dark contrast (top of the rings) indicates the presence of magnetic charge at the domain walls. Such a quasiuniform magnetization was also found in MFM studies of single micron-size permalloy rings. ${ }^{27}$ The magnetization (schematically shown by arrows) is aligned with the external field and all rings exhibit onion states, as in the simulations. At an applied field of -30 Oe [Fig. 11(b)], which is equal to the field value at which MOKE hysteresis loops show a large drop in signal (Fig. 6), several domain walls are nucleated and the structure is quite complex. However, the missing contrast inside of the right bottom ring indicates the existence of a vortex state. A small vortex is nucleated at the contact area between the top ring and the bottom left ring. Moreover, the top ring shows a uniform contrast, beside the small vortex core, suggesting a vortexlike configuration. Thus, the magnetization of the triring structure is similar to the $H_{y}$-vortex state predicted by the simulation, except that the geometrically equivalent bottom rings are permutated. Furthermore, in contrast to the simulation, the two rings in vortex states possess the same chirality of the magnetization, causing a contrast at the mutual boundary. While the tendency to form vortices is well predicted by the simulations, the calculated chiralities differ from the experiment. Although it may appear somewhat surprising that the remanent state observed in Fig. 11(b) is not the minimum energy state, similar observations have recently
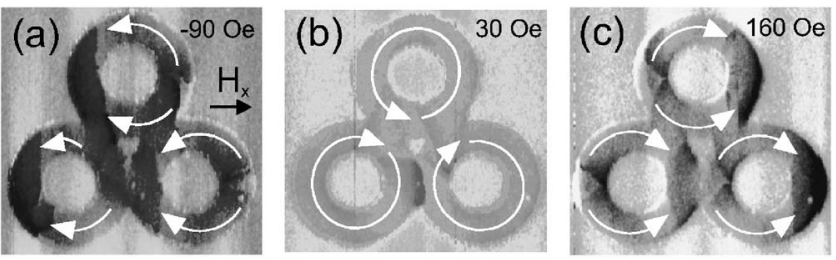

FIG. 12. MFM images of the reversal in an $H_{x}$ field. (a) At -90 Oe, the rings $(W=1 \mu \mathrm{m})$ are in an onion state. (b) At $30 \mathrm{Oe}$, the dark contrast indicates a domain wall between this two left rings while all three rings exhibit vortex states. (c) The vortices are annihilated and the rings form reverse onion states (160 Oe).

been reported for stripe domains in a Co bar where the remanent stripe domain state is shown not to be the lowest energy configuration. ${ }^{28}$ However, the field value of the switching into the quasi- $H_{y}$-vortex state found by MFM is smaller than that given by the simulations and MOKE. This is most likely due to calibration differences and/or the additional tip field.

With further increasing of field, the vortices are annihilated and reverse onion states form. At -160 Oe [Fig. 11(c)], the light and dark contrast can be assigned to the head-tohead domains associated with the reverse onion states.

In an $H_{x}$ field, the MFM images are consistent with a different reversal mechanism. Figure 12(a) shows the tri-ring structure at -90 Oe coming from negative saturation. The light (bottom) and dark (top) domains indicate onion states, but in contrast to the saturation field at -300 Oe (not shown here) the domains in the right ring show a deviation with respect to the direction of the applied field. These deviations likely lead to the small decrease of the signal in the MOKE hysteresis loops between -100 to 20 Oe. At 30 Oe [Fig. 12(b)], all rings switch into vortex states. This is comparable to the $\mathrm{B}^{\prime}$-vortex configuration predicted by the simulation (see Fig. 7). The vortex state of three rings is accompanied by magnetic frustration, but according to the simulations, the frustration is compensated by a small vortex core at the contact boundary of two of the rings. Instead of a vortex core, the MFM measurement shows the formation of a domain wall (dark contrast) between the rings. However, the experimental data are in better agreement with the $\mathrm{A}^{\prime}-\mathrm{B}^{\prime}-\mathrm{C}^{\prime}$ reversal type cycle, suggesting that this is the preferred reversal path. Likewise in MOKE (Fig. 7), the path followed by increasing the field from negative saturation exhibits better agreement between the experimental hysteresis and the simulation. At 160 Oe [Fig. 12(c)], the distinct domain wall between the rings disappears and each ring shows light and dark contrast, indicating reverse onion states.

\section{SUMMARY}

Interacting rings in direct contact arranged in a tri-ring structure exhibit a magnetic reversal process that is strongly affected by the direction of the external applied field as measured by MOKE. At remanence, all three rings yield an onion state, as in isolated rings. Reversal of the field leads to nucleation of vortices and vortex cores. However, the 
formation of vortices is frustrated due to the interactions between the rings within the tri-ring structure. Micromagnetic simulations reveal that this frustration can be resolved through several different vortex configurations. In particular, different intermediate magnetization states can be selected by changing the orientation of the applied field, some of which were experimentally verified by MFM. While the preferred reversal mode of vortex nucleation is determined by the angle of the applied field, the stability of the intermediate vortex states can be improved by decreasing the width of the rings.

\section{ACKNOWLEDGMENTS}

Work at Argonne is supported by the U.S. Department of Energy, Basic Energy Sciences-Materials Science under Contract No. W-31-109-ENG-38. The support of the International Student Exchange Program and the American Nuclear Society at Argonne National Laboratory is gratefully acknowledged (by V.R.), as is the U.S. National Science Foundation for Grants No. ECS-0202780 and DMR-0210519 (by V.M.) and the National Science and Engineering Research Consul of Canada (by K.B.).
*Corresponding author. Fax: +49 246161 3907; Email address: v.rose@fz-juelich.de

${ }^{1}$ R. P. Cowburn, J. Phys. D 33, R1 (2000).

${ }^{2}$ J. G. Zhu, Y. F. Zheng, and G. A. Prinz, J. Appl. Phys. 87, 6668 (2000).

${ }^{3}$ S. Y. Chou, P. R. Krauss, and L. S. Kong, J. Appl. Phys. 79, 6101 (1996).

${ }^{4}$ C. Ross, Annu. Rev. Mater. Res. 31, 203 (2001).

${ }^{5}$ K. J. Kirk, J. N. Chapman, S. McVitie, P. R. Aitchison, and C. D. W. Wilkinson, Appl. Phys. Lett. 75, 3683 (1999).

${ }^{6}$ Y. Hao, M. Walsh, M. Farhoud, C. A. Ross, H. I. Smith, J. Q. Wang, and L. Malkinski, IEEE Trans. Magn. 36, 2996 (2000).

${ }^{7}$ C. A. Ross, T. A. Savas, H. I. Smith, M. Hwang, and R. Chantrell, IEEE Trans. Magn. 35, 3781 (1999).

${ }^{8}$ J. Yu, U. Rüdiger, L. Thomas, S. S. P. Parkin, and A. D. Kent, J. Appl. Phys. 85, 5501 (1999).

${ }^{9}$ O. Fruchart, J. P. Nozieres, W. Wernsdorfer, D. Givord, F. Rousseaux, and D. Decanini, Phys. Rev. Lett. 82, 1305 (1999).

${ }^{10}$ Y. Zheng and J.-G. Zhu, J. Appl. Phys. 81, 5471 (1997).

${ }^{11}$ R. P. Cowburn, D. K. Koltsov, A. O. Adeyeye, M. E. Welland, and D. M. Tricker, Phys. Rev. Lett. 83, 1042 (1999).

${ }^{12}$ M. Kläui, C. A. F. Vaz, L. Lopez-Diaz, and J. A. C. Bland, J. Phys.: Condens. Matter 15, R985 (2003).

${ }^{13}$ J. Rothman, M. Kläui, L. Lopez-Diaz, C. A. F. Vaz, A. Bleloch, J. A. C. Bland, Z. Cui, and R. Speaks, Phys. Rev. Lett. 86, 1098 (2001).

${ }^{14}$ R. D. McMichael and M. J. Donahue, IEEE Trans. Magn. 33, 4167 (1997).

${ }^{15}$ M. Kläui, C. A. F. Vaz, J. A. C. Bland, L. J. Heyderman, F.
Nolting, A. Pavlovska, E. Bauer, S. Cherifi, S. Heun, and A. Locatelli, Appl. Phys. Lett. 85, 5637 (2004).

${ }^{16}$ M. Kläui, J. Rothman, L. Lopez-Diaz, C. A. F. Vaz, J. A. C. Bland, and Z. Cui, Appl. Phys. Lett. 78, 3268 (2001).

${ }^{17}$ M. Kläui, Adv. Solid State Phys. 44, 479 (2004).

${ }^{18}$ M. Kläui, C. A. F. Vaz, J. A. C. Bland, and L. J. Heyderman, Appl. Phys. Lett. 86, 032504 (2005).

${ }^{19}$ K. Y. Guslienko, V. Novosad, Y. Otani, H. Shima, and K. Fukamichi, Phys. Rev. B 65, 024414 (2002).

${ }^{20}$ U. Welp, V. K. Vlasko-Vlasov, G. W. Crabtree, J. Hiller, N. Zaluzec, V. Metlushko, and B. Ilic, J. Appl. Phys. 93, 7056 (2003).

${ }^{21}$ V. Novosad, K. Y. Guslienko, H. Shima, Y. Otani, S. G. Kim, K. Fukamichi, N. Kikuchi, O. Kitakami, and Y. Shimada, Phys. Rev. B 65, 060402 (2002).

${ }^{22}$ M. R. Scheinfein and Elizbeth A. Price, LLG Micromagnetics Simulator, 2003.

${ }^{23}$ L. D. Landau and E. M. Lifshitz, Phys. Z. Sowjetunion 8, 153 (1935).

${ }^{24}$ T. L. Gilbert, Phys. Rev. 100, 1243 (1955).

${ }^{25}$ M. Kläui, L. Lopez-Diaz, J. Rothman, C. A. F. Vaz, J. A. C. Bland, and Z. Cui, J. Magn. Magn. Mater. 240, 7 (2002).

${ }^{26}$ T. Okuda, H. Kiwata, T. Matsushima, T. Wakita, A. Harasawa, K. Ono, T. Kihara, M. Oshima, A. Yokoo, and T. Kinoshita, Jpn. J. Appl. Phys., Part 1 43, 4179 (2004).

${ }^{27}$ M. F. Lai, C. R. Chang, J. C. Wu, Z. H. Wei, J. H. Kuo, and J. Y. Lai, IEEE Trans. Magn. 38, 2550 (2002).

${ }^{28}$ G. Leaf, H. Kaper, M. Yan, V. Novosad, P. Vavassori, R. E. Camley, and M. Grimsditch, Phys. Rev. Lett. 96, 017201 (2006). 